\title{
Probing interstellar turbulence in cirrus with deep optical imaging: no sign of energy dissipation at $0.01 \mathrm{pc}$ scale
}

\author{
M.-A. Miville-Deschênes ${ }^{1}$, P.-A. Duc ${ }^{2}$, F. Marleau ${ }^{3}$, J.-C. Cuillandre ${ }^{2,4}$, P. Didelon ${ }^{2}$, S. Gwyn ${ }^{5}$, and E. Karabal ${ }^{2,6}$ \\ ${ }^{1}$ Institut d'Astrophysique Spatiale, CNRS, Univ. Paris-Sud, Université Paris-Saclay, Bât. 121, 91405 Orsay Cedex, France \\ e-mail: mamd@ias.u-psud.fr \\ 2 Laboratoire AIM, Paris-Saclay, CEA/IRFU/SAp - CNRS - Université Paris Diderot, 91191 Gif-sur-Yvette Cedex, France \\ 3 Institute of Astro and Particle Physics, University of Innsbruck, 6020 Innsbruck, Austria \\ 4 Observatoire de Paris, PSL Research University, 75014 Paris, France \\ 5 National Research Council of Canada, Herzberg Astronomy and Astrophysics Program, 5071 West Saanich Road, Victoria, BC, \\ V9E 2E7, Canada \\ ${ }^{6}$ European Southern Observatory, Karl-Schwarzschild-Str. 2, 85748 Garching, Germany
}

Received 14 March 2016 / Accepted 26 May 2016

\begin{abstract}
Diffuse Galactic light has been observed in the optical since the 1930s. We propose that, when observed in the optical with deep imaging surveys, it can be used as a tracer of the turbulent cascade in the diffuse interstellar medium (ISM), down to scales of about 1 arcsec. Here we present a power spectrum analysis of the dust column density of a diffuse cirrus at high Galactic latitude $\left(l \approx 198^{\circ}\right.$, $b \approx 32^{\circ}$ ) as derived from the combination of a MegaCam $g$-band image, obtained as part of the MATLAS large programme at the CFHT, with Planck radiance and WISE $12 \mu \mathrm{m}$ data. The combination of these three datasets have allowed us to compute the density power spectrum of the $\mathrm{HI}$ over scales of more than three orders of magnitude. We found that the density field is well described by a single power law over scales ranging from 0.01 to $50 \mathrm{pc}$. The exponent of the power spectrum, $\gamma=-2.9 \pm 0.1$, is compatible with what is expected for thermally bi-stable and turbulent H I. We did not find any steepening of the power spectrum at small scales indicating that the typical scale at which turbulent energy is dissipated in this medium is smaller than $0.01 \mathrm{pc}$. The ambipolar diffusion scenario that is usually proposed as the main dissipative agent, is consistent with our data only if the density of the cloud observed is higher than the typical values assumed for the cold neutral medium gas. We discuss the new avenue offered by deep optical imaging surveys for the study of the low density ISM structure and turbulence.
\end{abstract}

Key words. turbulence - methods: data analysis - techniques: high angular resolution - dust, extinction - ISM: structure local insterstellar matter

\section{Introduction}

In the early 20th century, optical images of star-forming regions revealed nebulae and dark regions indicating the presence of diffuse matter away from stars (Kapteyn 1909; Barnard 1910). Later, diffuse Galactic light (DGL) was observed in the optical away from the main star-forming complexes, first by Elvey \& Roach (1937) and then by de Vaucouleurs (1955, 1960), Lynds (1965). The DGL is mainly visible towards the Galactic plane but it has been noticed also at high Galactic latitudes (Sandage 1976). These early studies rapidly suggested that DGL could be the result of starlight scattered by diffuse matter spread out between stars, that is the interstellar medium (ISM). Later on, the ubiquity of the ISM was revealed by all-sky surveys at $21 \mathrm{~cm}$ and in the infrared that showed that there is no line of sight on the sky without interstellar matter.

Following the detection of the infrared emission at high Galactic latitude with IRAS (Low et al. 1984), there have been several studies showing the good spatial correlation between the optical DGL and the infrared emission, especially at $100 \mu \mathrm{m}$ (de Vries \& Le Poole 1985; Laureijs et al. 1987; Guhathakurta \& Tyson 1989; Paley et al. 1991; Zagury et al. 1999; Ienaka et al. 2013). The strong spatial correlation with the infrared and the spectral energy distribution of the DGL ${ }^{1}$ confirmed that it is caused by scattering by large dust grains (Brandt \& Draine 2012).

For extra-galactic studies, one obvious consequence of the presence of dust in the diffuse ISM, referred to as cirrus, is that light emitted in galaxies is partly absorbed and scattered by interstellar dust along the way, even in diffuse areas of the sky. Moreover, dust does not only produce a chromatic extinction of extra-galactic light, it also scatters photons of the interstellar radiation field towards the observer (the DGL) adding up some brightness to the extra-galactic emission. This is recognized as a nuisance for the study of the diffuse emission in galaxy halos and in between galaxies using deep optical imagery (Cortese et al. 2010; Duc et al. 2015).

Interstellar dust might be a nuisance for extra-galactic studies but, in principle, DGL could also be a useful tracer of interstellar column density, complementing far-infrared and sub-millimeter dust emission (Juvela et al. 2006). In the past decades, to understand the details of the star-formation process, the focus has been on denser and more massive environments like molecular clouds. For denser regions, the interpretation of scattered light becomes

1 The DGL is also observed in the UV (Boissier et al. 2015, and references therein). 
difficult as the observed structure depends on the properties of grains and on the geometry of the radiation field (Zagury et al. 1999). In practice, the UV-visible part of the radiation field gets absorbed efficiently as the column density increases. For studying molecular clouds, near-infrared observation was preferred to optical because of the lower opacity. Nevertheless, the interpretation of near-infrared DGL still requires the use of radiative transfer modeling to trace structure and study the evolution of dust properties (Malinen et al. 2012, 2013).

Radiative transfer effects are much less of a concern when looking at diffuse regions of the sky, even in the optical. We notice that optical DGL has not been exploited yet as a way to quantify the structure of the diffuse ISM, even though the large field of view cameras now equipping four-to-eight meter class telescopes offer mapping capabilities with degree size fields of view and with arcsecond angular resolution. It is difficult to access such a large dynamical range in scales with other observational probes. One potentially interesting application of such high angular resolution observations is to be able to study the properties of interstellar turbulence down to scales close to the dissipation scale.

The formation of dense interstellar structures, where stars form, depends strongly on the way energy and mass is transferred through scales. By creating an energy cascade through more than four orders of magnitude in scale and by producing density fluctuations, interstellar turbulence is a key agent of the overall star-formation process. The turbulent cascade of the ISM is more complex than purely hydrodynamical turbulence as it involves compressibility, gravity, thermal instability and magnetic fields which are important elements of the dynamics of the ISM. Polarization observations, in the radio from synchrotron and in the visible-infrared-submm from dust, show a link between the structure of the ISM and the morphology of the magnetic field (Planck Collaboration Int. XIX 2015; Planck Collaboration Int. XX 2015; Planck Collaboration Int. XXXII 2016; Planck Collaboration Int. XXXIII 2016; Planck Collaboration Int. XXXV 2016). The Planck observations are compatible with one expected behavior in which matter flows along the field lines, where there is less resistance (Soler et al. 2013). In the diffuse ISM, matter is seen to be organized parallel to the field lines while dense and gravitational bound filaments are more perpendicular to the field lines.

The magnetic field is involved in the structure of matter but it is likely that it is also playing a dominant role in the dissipation of the turbulent energy. As the ISM is partly ionized, even in the dense regions, there is friction between the ions and neutrals known as the ambipolar diffusion. This process dissipates energy at a scale larger than the more classical molecular viscosity. Recently the analysis of Herschel observations have shown that the main filaments of several close-by molecular clouds have a typical width of $0.1 \mathrm{pc}$ irrespective of their distance and of their column density (Arzoumanian et al. 2011). Hennebelle (2013) and Hennebelle \& André (2013) suggested that this might be the signature of the dissipation of turbulent energy by ion-neutral friction. Alternative explanations have been proposed, for example by Fischera \& Martin (2012) who suggested that $0.1 \mathrm{pc}$ is a natural scale for a self-gravitating filament in pressure equilibrium with its surrounding. It is important to note that even if the typical scale found in filaments of molecular clouds might be related to the dissipation of turbulent energy, such a scale has not been identified yet in any spectral analysis of the gas or dust column density (e.g., see Miville-Deschênes et al. 2010).

Here we propose to use the DGL to study the structure of the diffuse ISM where gravity does not play a dominant role in the dynamics. One advantage of looking at diffuse areas of the sky, away from star-forming regions, is that dust is uniformly heated by the interstellar radiation field, limiting the effects of radiative transfer in the observed structure. We suggest that large optical telescopes equipped with large field of view camera provide a unique opportunity to study the properties of the interstellar turbulence cascade by observing close-by clouds. For example, in good seeing conditions, the MegaCam instrument on the CFH telescope can achieve an angular resolution of 0.5 arcsec. For clouds at a distance of $100 \mathrm{pc}$, typical for clouds in the solar neighborhood, this corresponds to a linear physical scale of $2 \times$ $10^{-4} \mathrm{pc}$, or about $50 \mathrm{AU}$. The use of such observations opens a new perspective on the study of the small-scale structure of the ISM and potentially on the identification of the dissipative processes at play.

In this paper we present CFHT/MegaCam observations of a diffuse region at high Galactic latitude. These data are complemented by Planck and WISE data in order to estimate the power spectrum of density fluctuations of the diffuse ISM. The paper is organized as follow. In Sect. 2 we present the data used in this study. Section 3 presents the power spectrum analysis. We discuss our results in Sect. 4.

\section{Observing interstellar dust with large optical telescope}

The processing of optical observations of external galaxies is traditionally done in such a way that emission at angular scales larger than the typical size of galaxies is removed, in order to reject spatial variation of the sky background. By doing this, most of the DGL is filtered out. Furthermore, images suffer from residual instrumental scattered light. However a substantial gain in the ability of restoring the diffuse emission on large angular scales was obtained using ultra deep and long exposures on amateur-type small telescopes (Martínez-Delgado et al. 2010) or dedicated observing strategies on large telescopes and processing the data with optimized pipelines such as Elixir-LSB (Ferrarese et al. 2012; Duc et al. 2015). These efforts were initially motivated by the detection around nearby galaxies of low surface-brightness collisional debris such as tidal tails. At the surface brightness limit reached by these surveys (typically around $29 \mathrm{mag} \mathrm{arcsec}^{-2}$ ), images are often polluted by extended patchy diffuse emission from Galactic cirrus. Here we exploit one such image, obtained as part of the MATLAS project, a large program with the MegaCam camera installed at the Canada France Hawaii Telescope, aimed at obtaining deep multi-band images of a complete sample of early-type galaxies from the ATLAS $^{3 \mathrm{D}}$ sample (Cappellari et al. 2011). A dithering method with large offsets was used. Final images consist of stacks of seven individual images, with total exposure time of $40 \mathrm{~min}$ (Duc et al. 2015). The target is the field of the galaxies NGC 2592 and NGC 2594, located at $l=197.9^{\circ}, b=32.2^{\circ}$. The $g$-band map of this field is shown in Fig. 1. The angular resolution of this image is $1.3^{\prime \prime}$ and field of view $1.04^{\circ} \times 1.15^{\circ}$. It shows prominent filamentary but mostly patchy diffuse emission with structures at all scales.

Duc et al. (2015) already identified the very good match between the diffuse emission of the NGC 2592-2594 field and the dust emission detected by Planck-HFI at $857 \mathrm{GHz}(350 \mu \mathrm{m})$, which leaves little doubt on the DGL nature of the observed filaments. As shown in Fig. 1, the correspondence is also very good with the radiance from Planck (Planck Collaboration XI 2014) and with the WISE $12 \mu \mathrm{m}$ emission (Meisner \& Finkbeiner 2014). 

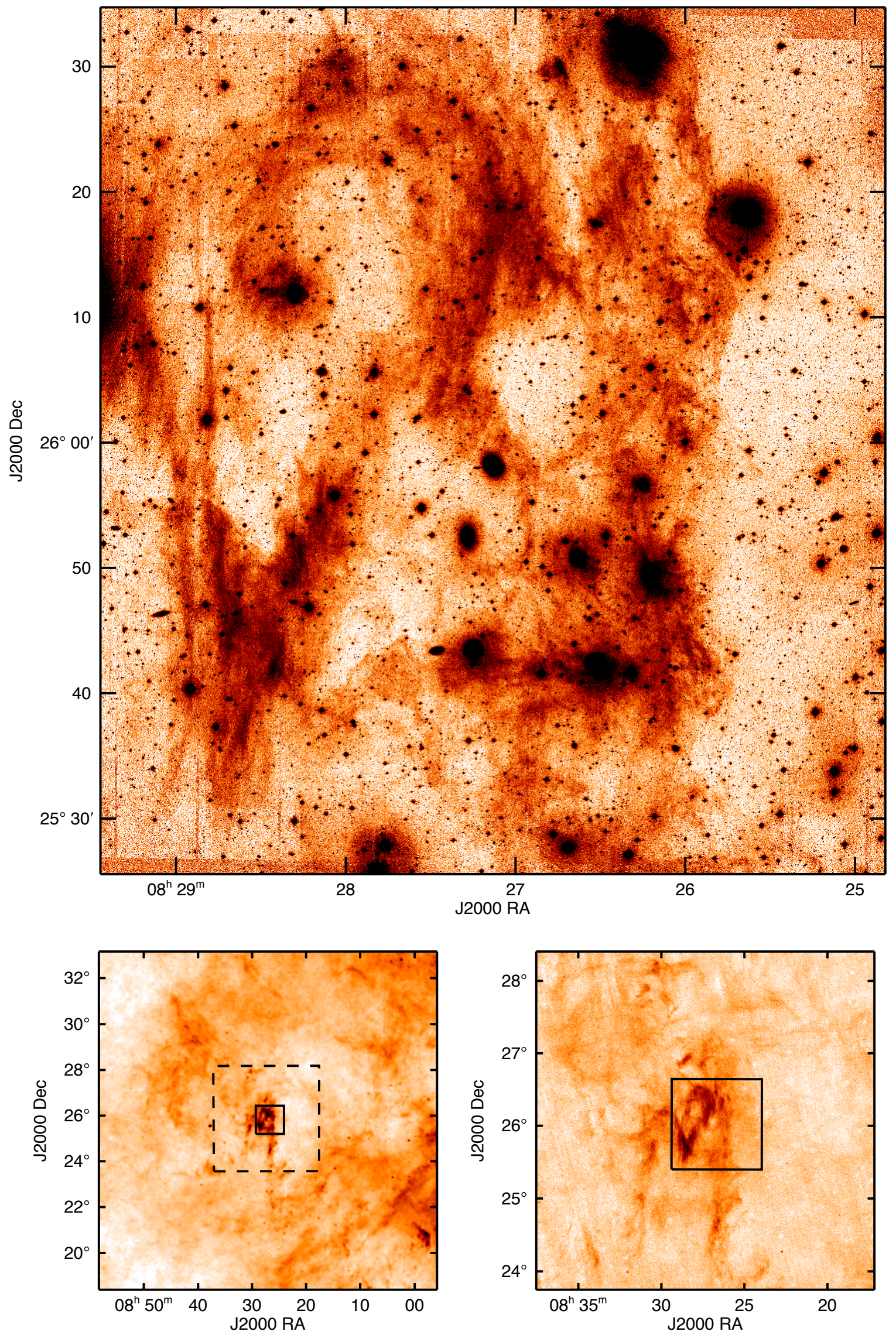

Fig. 1. Top: MegaCam $g$-band image of the field around NGC 2592 and NGC 2594. The two galaxies are located close to the center of the image. Bottom-left: Planck radiance. Bottom-right: WISE $12 \mu \mathrm{m}$. The black rectangle indicates the area of the MegaCam field. The dashed-line rectangle on the radiance map indicates the area of the WISE field shown on the right. 
The radiance map provided by Planck Collaboration XI (2014) has an angular resolution of $5^{\prime}$. It is thought to be the best estimate of dust column density in the diffuse areas of the sky, where $E(B-V)<0.3$ (or $A_{\mathrm{V}}<0.93$ ) and where the interstellar radiation field intensity is uniform, which is the case here. The main advantage of the radiance is that it provides a way to limit the impact of the cosmic infrared background (CIB) anisotropies that degrade strongly the measure of dust optical depth at a given frequency (see Planck Collaboration XI 2014 for details). The main structures seen in the MegaCam $g$-band image are also visible in the radiance despite the lower angular resolution ${ }^{2}$. The radiance, shown in Fig. 1 for a $15^{\circ} \times 15^{\circ}$ region centered on NGC 2592, reveals the extended diffuse emission in the region highlighting the fact that the main structure seen with MegaCAM is slightly brighter than average.

The WISE $12 \mu \mathrm{m}$ map reprocessed by Meisner \& Finkbeiner (2014) has an angular resolution of $15^{\prime \prime}$, intermediate between Planck and MegaCam. A $4.7^{\circ} \times 4.7^{\circ}$ region centered on NGC 2592 is shown in Fig. 1. The $12 \mu \mathrm{m}$ emission is dominated by the emission from the smallest dust grains (often attributed to polycyclic aromatic hydrocarbons - PAHs). It has been shown that the abundance of these small dust grains evolves rapidely in the ISM through fragmentation and coagulation processes (Miville-Deschênes et al. 2002a). In molecular clouds, the emission from these small dust grains tends to be lower, probably because they stick to bigger grains (Stepnik et al. 2003). Nevertheless, in a uniformly illuminated region of relatively small volume density, like the high Galactic cloud studied here, it is likely that the PAH emission is proportional to the gas column density, at least to first order. In that respect, the excellent spatial correspondance of the WISE map with the radiance from Planck seen in Fig. 1 is reassuring. It is important to mention that in their reprocessing of the WISE $12 \mu \mathrm{m}$ map, Meisner \& Finkbeiner (2014) used the Planck $857 \mathrm{GHz}$ data to recover the emission at scales larger than $2^{\circ}$.

\section{Power spectrum of column density}

\subsection{Context}

One way of exploring the turbulent cascade and the associated dissipative processes is to study the statistical properties of column density fluctuations of interstellar clouds. In particular the power spectrum of column density is bearing information about the way density is organized in three dimensions and therefore about the very nature of the turbulent flow. Several studies showed that the power spectrum of the column density of an optically thin tracer is equal to the power spectrum of the density (e.g., Miville-Deschênes et al. 2003). If the power spectrum of the column density provides some information on the inertial range of the turbulence and on the type of the turbulent flow, it is plausible that the dissipation of the turbulent energy will also leave an imprint. Typically, the dissipation of the turbulent energy induces a loss of power in the velocity field at scales of the order of the dissipation scale. Because density and velocity fluctuations are related, it is expected that the power spectrum of the density field (and therefore of the column density field) will show a similar loss of power at small scales. This effect is clearly seen by Ntormousi et al. (2016) in their analysis of numerical simulations dedicated to the study of the imprint of ambipolar diffusion on the statistical properties of the diffuse ISM.

\footnotetext{
2 The angular resolution of MegaCam is about 300 times better than that of Planck.
}

Based on this idea, the present study focuses on the characterization of the power spectrum of column density fluctuations of the NGC 2592/2594 field. In principle, by combining the MegaCam data with Planck and WISE data, we are able to study the statistical properties of the density fluctuations on scales ranging from $\sim 1^{\prime \prime}$ to $15^{\circ}$. Obviously, the combination of these three data sets can not be done everywhere on the sky as they trace dust in different ways: the big grain emission (Planck), the PAH emission (WISE) and the scattering from big grains (MegaCAM). In the very diffuse area of the sky studied here, where there is no local heating source and where the column density is in a range where radiative transfer and dust evolution effects are minimal, these three observations are assumed to be reliable tracers of the dust and gas column density.

\subsection{Star and galaxy removal}

One obvious aspect of the $g$-band data is the presence of a large number of point-like sources, that is, stars and distant galaxies, and extended nearby galaxies, including NGC 2592/2594. These sources dominate the power in the map. They must be removed in order to study the power spectrum of the Galactic diffuse emission. To do so, we used initial catalogs of individual sources computed by SExtractor (Bertin \& Arnouts 1996), using different values of the isoarea_image parameter. The affected pixels were then replaced by a median value of the surrounding background. In the process, $8.7 \%$ of pixels were modified.

Figure 1 shows the quality of the MegaCam data processing. In this image, the identified individual sources that were masked in the final image appear as black. The footprint of the individual MegaCam images is barely visible. The vertical lines at the top and bottom of the image are part of a residual instrumental signature. Apart from them, all features visible in the image are real, including the multiple very narrow filaments. The smallscale structure of the DGL is thus revealed with striking details.

\subsection{Power spectrum analysis}

The power spectrum of the three maps (MegaCam, WISE and Planck radiance) was computed using a standard technique (see Miville-Deschênes et al. 2002b, 2010). The power spectrum, $P(k)$, is the azimuthal average of the modulus of the Fourier transform of each image. To avoid edge effects in the $X$ and $Y$ direction due to the replication of the image done by the fast Fourier transform algorithm, the edges of the images were first slightly apodized using a cosine function. This has almost no effect on the result as the three maps show very small large scale gradients.

The power spectrum of each map was modeled using the expression

$$
P(k)=B(k) \times\left[A k^{\gamma}+C k^{\beta}\right] .
$$

Here we assumed that the power spectrum of each map is well represented by two components convolved by a Gaussian beam, $B(k)$. The first contribution to the power spectrum, $A k^{\gamma}$, is the interstellar dust that is assumed to follow a power law. The second contribution, $C k^{\beta}$, is included to represent the contribution from point sources and from the noise. In this type of analysis, this second component is often assumed to be white $(\beta=0)$ but it can also be gray $(\beta<0)$ depending on the statistical properties of the noise, potential instrumental effects or residual contamination emission ( $1 / \mathrm{f}$ noise has $\beta=-1$ ). The 

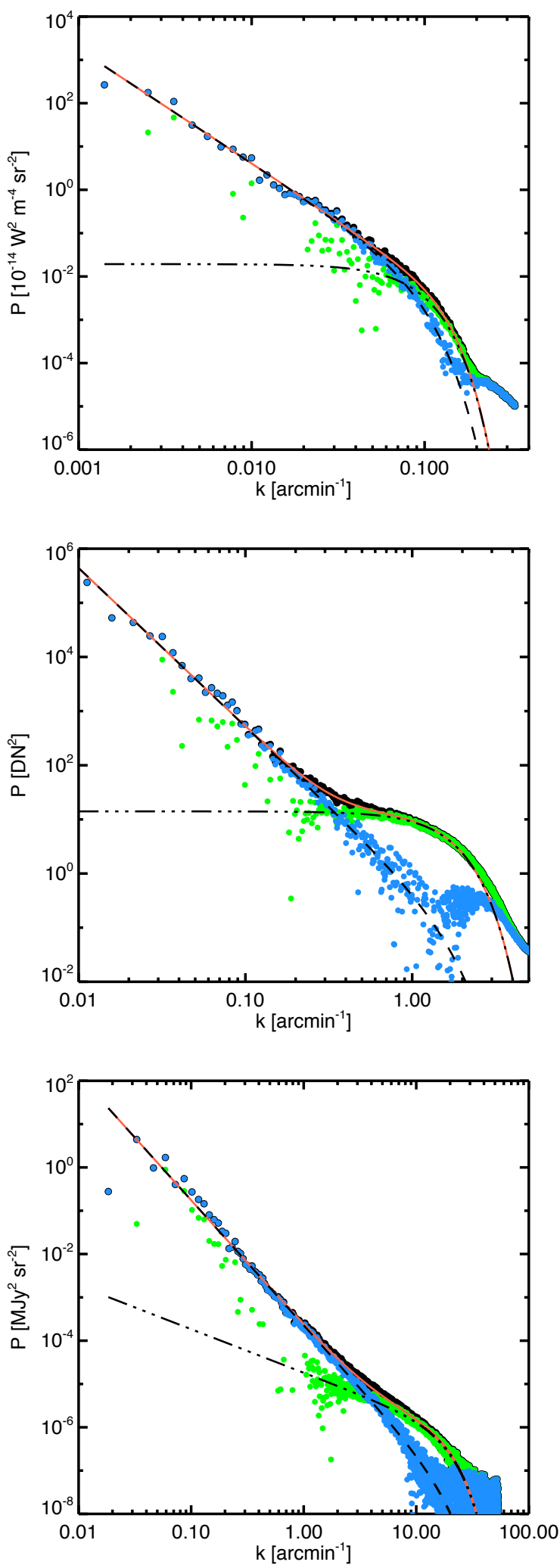

Fig. 2. Power spectrum model of the individual maps: Planck (top), WISE (middle) and MegaCam (bottom). The power spectrum is given by the black dots. The global fit is the red curve. The blue points represent the cirrus component $\left(P(k)-B(k) \times C k^{\beta}\right)$ while the green dots represent the noise component $\left(P(k)-B(k) \times A k^{\gamma}\right)$. The dashed and dash-dotted curves are the results of the fit for the cirrus and noise terms respectively. The slope of the dust emission power spectrum is $\gamma=-2.7 \pm 0.1, \gamma=-2.9 \pm 0.1$ and $\gamma=-2.9 \pm 0.1$ for Planck, WISE and MegaCam, respectively.

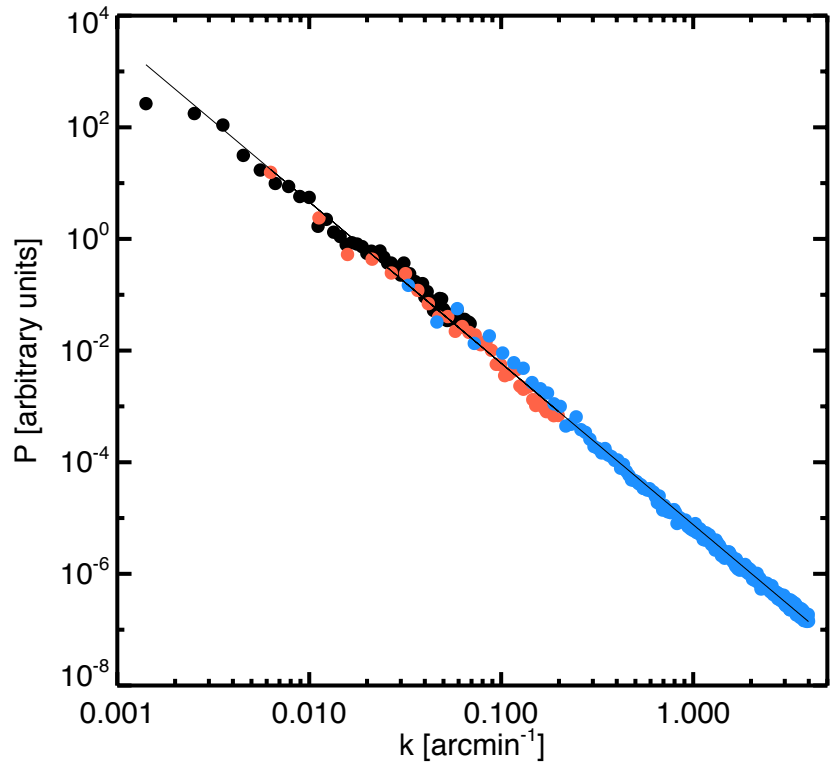

Fig. 3. Combined power spectrum showing the three $P(k)$ : black is Planck radiance, red is WISE and blue is MegaCam. The units of the $y$ axis are arbitrary; each $P(k)$ was scaled in order to match the others. For each $P(k)$, we show data points corresponding to scales larger than the beam and where the power is above the noise component. The data points shown here are noise subtracted and divided by the beam function. The best fit gives $P(k) \propto k^{-2.9 \pm 0.1}$.

FWHM of the Gaussian beam was set to $5^{\prime}, 15^{\prime \prime}$ and $1.3^{\prime \prime}$ for Planck, WISE and MegaCam respectively.

The power spectrum of each map is shown in Fig. 2 (top Planck radiance, middle WISE, bottom MegaCam). For each panel, the black points represent the power spectrum of each map. The global fit is the red curve. The cirrus (blue points and dashed curve) and noise components (green points and dash-dotdot-dot curve) are also shown.

The three maps have a power spectrum that is well described by a power law at large angular scales (low values of $k$ ). The slope of the cirrus component is almost the same in the three maps: $\gamma=-2.7 \pm 0.1, \gamma=-2.9 \pm 0.1$ and $\gamma=-2.9 \pm 0.1$ for Planck, WISE and MegaCam respectively.

For Planck and WISE, the noise term is well fit with $\beta=0$, compatible with white noise or with CIB anisotropies. This term is extremely small for Planck in accordance with the fact that CIB anisotropies are small in the radiance map. For WISE the noise term is significant; it reveals the limited signal-to-noise ratio of the data in such diffuse areas of the $\mathrm{sky}^{3}$. Contrary to the two other maps, the noise term of MegaCam is not white. The best fit is compatible with $\beta=-1.01$ ( $1 / \mathrm{f}$ noise). The structure in the noise is likely dominated by residual emission from individual sources removal.

The similarities in the cirrus power spectrum for the three maps are better seen in Fig. 3 where they are combined in the same plot. Here the units are arbitrary; each $P(k)$ was scaled in order to match the others on the common scales. For each $P(k)$, we show only the data points corresponding to scales larger than the beam and where the power is above the noise component.

3 Meisner \& Finkbeiner (2014) convolved the data and thus the noise to $15^{\prime \prime}$ resolution. 


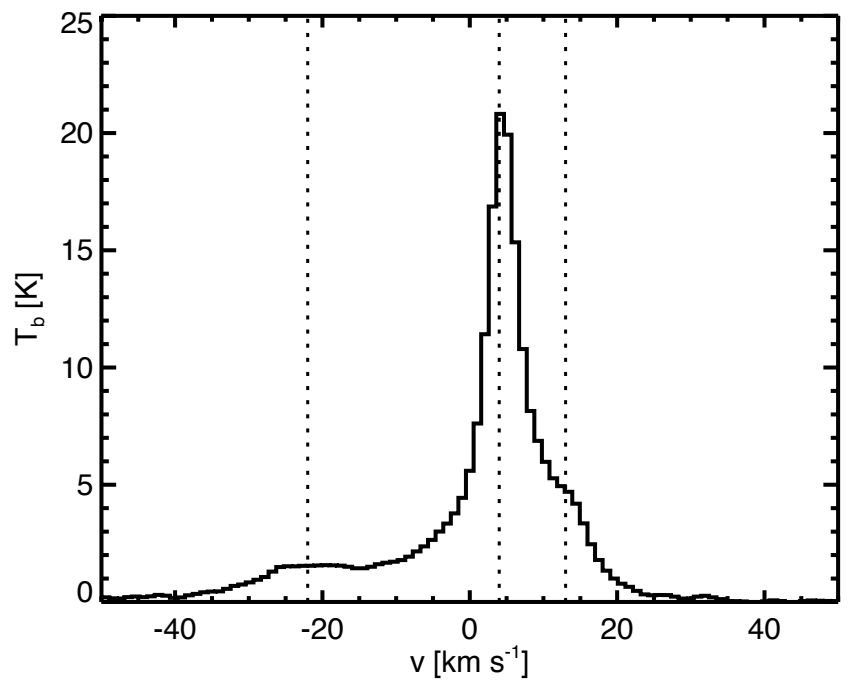

Fig. 4. $21 \mathrm{~cm}$ spectrum at the central position of the NGC 2592 ATLAS field. Data are from the LAB survey (Kalberla et al. 2005). The dashed lines $\left(v_{\mathrm{LSR}}=-21.6,4.1\right.$ and $\left.13.4 \mathrm{~km} \mathrm{~s}^{-1}\right)$ correspond to the channel maps shown in Fig. 6.

\section{Discussion}

\subsection{Where is the cirrus optical emission coming from?}

Before discussing the results of the power spectrum analysis, it is important to estimate the distance to the extended diffuse structure observed with MegaCam. To our knowledge, there is no direct measure of distance to the specific cirrus structure studied here. Estimating the exact distance to the cloud is difficult without absorption measurements of stars located behind it. According to the 3D map of the dense clouds around the Sun produced by Lallement et al. (2014), there is very little dense material in the direction of NGC 2592-2594 $\left(l=197.9^{\circ}\right.$, $\left.b=32.2^{\circ}\right)$. It is therefore difficult to accurately estimate the distance to the cloud.

Given that the field is at high Galactic latitude $\left(b=31.6^{\circ}\right)$, one possibility could be that the structure observed is part of the Galactic halo. In that case it is probable that it would fall into the Intermediate-Velocity range. To evaluate this we looked at $21 \mathrm{~cm}$ from the Leiden/Argentine/Bonn (LAB) survey (Kalberla et al. 2005). Even with its coarse angular resolution of $30^{\prime}$, the average velocity of the $21 \mathrm{~cm}$ emission provides a mean to identify if the emission is coming from the local ISM or if it is located in the Galactic halo. Figure 4 shows the $21 \mathrm{~cm}$ emission spectrum at the central position of the MegaCam field. It reaches $T_{b} \approx$ $21 \mathrm{~K}$ and the $\mathrm{H}$ I column density is $N_{\mathrm{HI}}=4.3 \times 10^{20} \mathrm{~cm}^{-2}$, both estimated within a $30^{\prime}$ beam. The main structure seen in the dust tracers (MegaCam, WISE and Planck) is seen at a $21 \mathrm{~cm}$ velocity of about $4 \mathrm{~km} \mathrm{~s}^{-1}$ (Fig. 6, middle). Therefore it is unlikely to be located in the Galactic halo where clouds usually have negative velocities as they slow down when entering the disk.

The high Galactic latitude and the fact that it is seen at $21 \mathrm{~cm}$ as a narrow cold neutral medium (CNM) component at a LSR velocity close to zero, are indications that the observed structure is in the solar neighborhood. It is a fairly compact structure, less than one degree across. It could be a small, dense cloud that was not identified in the 3D map of Lallement et al. (2014) based on a limited number of sightlines to background stars. We will make the assumption that the cloud is likely to be located within the thin H I disk of $H W H M=106$ pc (Dickey \& Lockman 1990). At

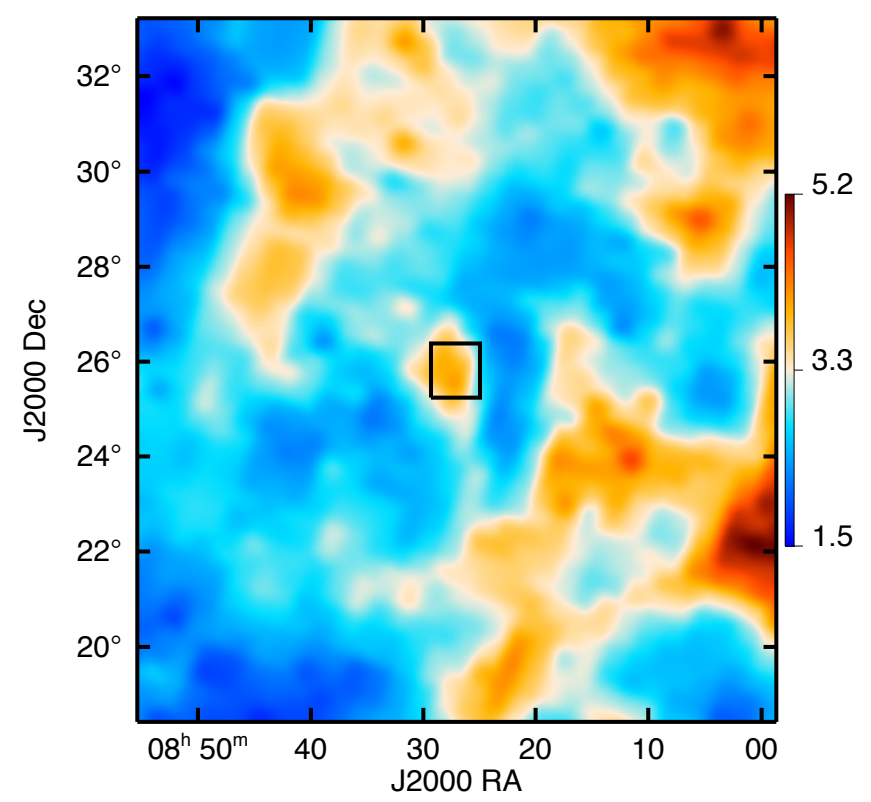

Fig. 5. Map of the H I column density estimated from the $21 \mathrm{~cm}$ data of the LAB survey (Kalberla et al. 2005). The units are $10^{20} \mathrm{~cm}^{-2}$. The area shown is the same as for the Planck radiance (Fig. 1). The black square indicates the ATLAS field.

the latitude of the cloud, this translates into a probable distance of $200 \mathrm{pc}$, a value we adopt here.

\subsection{The power spectrum of density fluctuations}

The combined power spectrum presented in Fig. 3 indicates that the power spectrum of density fluctuations in cirrus follows a power law with a constant exponent, $\gamma=-2.9 \pm 0.1$, over three orders of magnitude in scales. Our analysis does not reveal any characteristic scale, neither at large nor small scales. The exponent found here is comparable to what was found earlier using $100 \mu \mathrm{m}$ IRAS data, $\gamma \sim-2.9$, (Gautier et al. 1992; Miville-Deschênes et al. 2007) or $250 \mu \mathrm{m}$ Herschel-SPIRE data on a similar range of scales (down to $30^{\prime \prime}$ ), $\gamma=-2.7 \pm 0.1$, (Miville-Deschênes et al. 2010). Our results are also similar to what was found recently using $21 \mathrm{~cm}$ observations of diffuse high Galactic latitude fields (values ranging from -2.5 to -3.0 ) by Martin et al. (2015) and Blagrave et al. (2016).

Even though no characteristic scale is found in the power spectrum, the exact value of $\gamma$ provides some information about the properties of interstellar turbulence. For compressible, isothermal and subsonic turbulence, Kim \& Ryu (2005), Kritsuk et al. (2007), Saury et al. (2014) showed that the power spectrum of density follows that of the velocity $\left(P_{k}(n) \sim k^{-11 / 3}\right)$. This would produce a column density power spectrum with $\gamma=-3.7$, far from what is observed. For compressible turbulence, the increase of the Mach number into the supersonic regime leads to larger small-scale density fluctuations and therefore to a flatter power spectrum of the (column) density. According to Kim \& Ryu (2005), a density power spectrum with $\gamma=-2.9$ would correspond to an isothermal flow with a Mach number $M \sim 7$.

Similarly a flatter power spectrum is also observed in the case of thermally bi-stable gas (Gazol \& Kim 2010) even when the turbulence is subsonic (Saury et al. 2014). In particular, Saury et al. (2014) showed that the thermal instability generates high density contrasts, without having to rely on the effect 

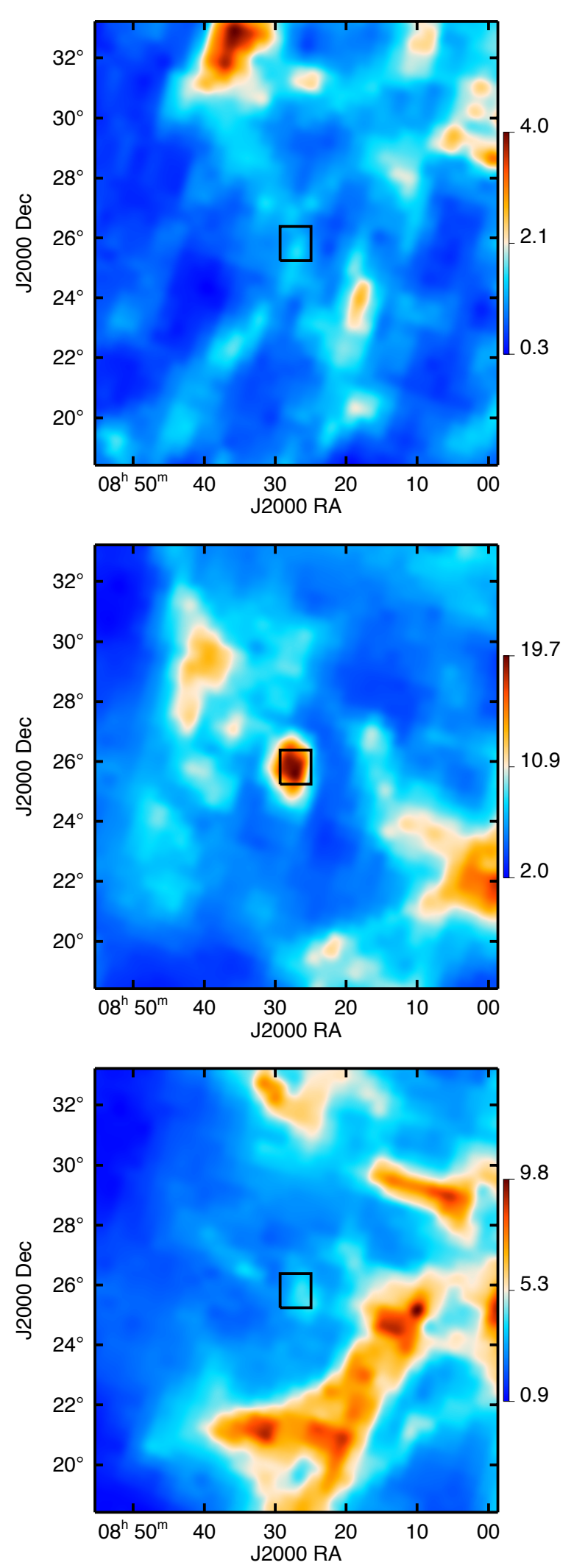

Fig. 6. Channel maps of $21 \mathrm{~cm}$ brightness temperature for the NGC 2592 area. Data are from the LAB survey (Kalberla et al. 2005). Units are $\mathrm{K}$. The three channels maps are for $v_{\mathrm{LSR}}=-21.6 \mathrm{~km} \mathrm{~s}^{-1}$ (top), $4.1 \mathrm{~km} \mathrm{~s}^{-1}$ (middle) and $13.4 \mathrm{~km} \mathrm{~s}^{-1}$ (bottom). They correspond to the dashed lines of Fig. 4. We note the different dynamic range of each map. The area shown is the same as for the Planck radiance (Fig. 1). The black square indicates the MegaCam field. of supersonic turbulence. In their simulation they showed that for typical properties of the WNM in the solar neighborhood, the density field of the H I (CNM plus WNM) has a power law power spectum with $\gamma \sim-2.5$.

The $21 \mathrm{~cm}$ data in this area of the sky is typical of high Galactic latitude cirrus. The $21 \mathrm{~cm}$ line emission (Fig. 4) is characterized by a narrow component $\left(F W H M \sim 5 \mathrm{~km} \mathrm{~s}^{-1}\right)$ on top of a wide one $\left(F W H M \sim 20 \mathrm{~km} \mathrm{~s}^{-1}\right)$, a classical picture of the thermally bi-stable HI. This is compatible with the fact that the density structure is the result of thermal instability of the H I.

\subsection{The ion-neutral friction scale}

The structure observed with MegaCam is likely to be a mixture of CNM and WNM located in the solar neighborhood. The power spectrum analysis shown in Sect. 3.3 reveals that the cirrus density structure is characterized by a single power law over the whole range of scales. Assuming that turbulence is involved in shaping the multi-scale structure of the density field, it implies that our analysis did not reach the energy injection scale nor the dissipation scale. In this context, it is interesting to evaluate what is the smallest physical scale probed by the MegaCam data. Given the presence of a noise component at small angular scale (Fig. 2-bottom), the scale at which the cirrus and noise components are equal is $k \sim 4 \operatorname{arcmin}^{-1}$, corresponding to $15^{\prime \prime}$. Assuming a distance to the cloud of $200 \mathrm{pc}$, the smallest accessible scale is $0.01 \mathrm{pc}$.

In the ISM, two processes are considered for dissipating the turbulent energy: molecular dissipation and ambipolar diffusion. Given the low density of the ISM and the fact that is never totally neutral, it is likely that the dissipation of the turbulent energy is dominated by ion-neutral friction (ambipolar diffusion). Following Lequeux (2005), Miville-Deschênes et al. (2016) the scale at which ambipolar diffusion becomes significant for a partially ionized medium is given by

$l_{\mathrm{AD}}=\sqrt{\frac{\pi}{\mu_{n}}} \frac{B}{2 X\langle\sigma v\rangle n_{n}^{3 / 2}}$,

where $X$ is the ionization fraction, $n_{n}$ the density of neutrals, $\langle\sigma v\rangle$ the collision rate between ions and neutral (assumed to be the Langevin rate $2 \times 10^{-9} \mathrm{~cm}^{3} \mathrm{~s}^{-1}$ ) and $\mu_{n}$ the molecular weight of the neutrals, taken to be $1.4 m_{\mathrm{p}}$ where $m_{\mathrm{p}}$ is the proton mass.

The difficulty in estimating $l_{\mathrm{AD}}$ is in defining typical values for $n_{n}, B$ and $X$ as a function of environment or phases. As summarized by Heiles \& Crutcher (2005), the total field strength in CNM clouds in the solar neighborhood is, on average, $B_{\text {tot }}=6.0 \pm 1.8 \mu \mathrm{G}$. This is also the average field strength value measured in the diffuse warm components of the ISM (WIM, WNM) using synchrotron data (Beck 2001). It is therefore assumed that the magnetic field strength does not depend significantly on gas density for $n<100 \mathrm{~cm}^{-3}$. At higher densities, in molecular clouds, the field strength is observed to scale roughly with the square root of density with $\left.B \approx B_{0} \sqrt{(} n / n_{0}\right)$ with $B_{0}=$ $25 \mu \mathrm{G}$ and $n_{0}=10^{3} \mathrm{~cm}^{-3}$ (Crutcher 1999).

In the cold $\mathrm{HI}$, the ionization fraction (or equivalently the number of free electrons) is given by the abundance of ionized carbon (CII) which is the main source of electrons. It is close to $X=1 \times 10^{-4}$ (Wolfire et al. 2003). In the warm H I the ionization fraction increases to $3 \times 10^{-3}$. In molecular clouds $X$ depends strongly on local conditions; it is assumed to be in the range $X=10^{-6}-10^{-7}$ (Bergin \& Tafalla 2007).

The density of the molecular gas varies greatly, depending on scale and mass of the clouds, with values ranging from $10^{2}$ to 
Table 1. Typical dissipation scales of ambipolar diffusion for different interstellar conditions.

\begin{tabular}{ccccc}
\hline \hline Medium & $\begin{array}{c}n_{n} \\
{\left[\mathrm{~cm}^{-3}\right]}\end{array}$ & $\begin{array}{c}B \\
{[\mu \mathrm{G}]}\end{array}$ & $X$ & $\begin{array}{c}l_{\mathrm{AD}} \\
{[\mathrm{pc}]}\end{array}$ \\
\hline Molecular clouds & $10^{4}$ & 80 & $1 \times 10^{-7}$ & 0.1 \\
Cold H I & 40 & 6 & $1 \times 10^{-4}$ & 0.02 \\
Warm H I & 0.5 & 6 & $3 \times 10^{-3}$ & 0.5 \\
\hline
\end{tabular}

$10^{6} \mathrm{~cm}^{-3}$ (Ferrière 2001). Here we assume $n=10^{4} \mathrm{~cm}^{-3}$. The $\mathrm{HI}$ average density is better constrained as it is mostly set by the pressure equilibrium of the two-phase medium (Wolfire et al. 2003; Saury et al. 2014). Here we assume $n(\mathrm{CNM})=40 \mathrm{~cm}^{-3}$ and $n(\mathrm{WNM})=0.5 \mathrm{~cm}^{-3}$.

Given these physical conditions for molecular clouds, CNM and WNM (summarized in Table 1), and using Eq. (2), the ambipolar diffusion scales for the three media are $0.1 \mathrm{pc}, 0.02 \mathrm{pc}$ and $0.5 \mathrm{pc}$, respectively. The ion-friction scale is the smallest in the cold H I but the difference with the molecular phase is not immense considering the very different physical conditions. This is explained by the specific dependance of $l_{\mathrm{AD}}$ on $n_{n}, B$ and $X$ (see Eq. (2)); the lower density of the CNM and lower magnetic field strength are almost compensated by the higher ionization fraction. Interestingly, it is in the warmer phase, where the ionization fraction is the highest, that the ion-neutral friction scale is the largest.

The relatively narrow $21 \mathrm{~cm}$ spectral feature (Fig. 4) associated with the cloud seen in the $g$-band image indicates that a significant fraction of the gas is in the CNM phase. We estimate that for density and magnetic field intensity typical of the CNM, the ion-neutral friction scale is about $0.02 \mathrm{pc}$. At a distance of $200 \mathrm{pc}$ this corresponds to 20 arcsec (or equivalently to $2.9 \operatorname{arcmin}^{-1}$ ). This corresponds to an angular scale where the signal-to-noise ratio of the MegaCam data is slightly above one (Fig. 2) but no steepening of the power spectrum is observed. We conclude that the CNM dissipation scale is smaller than 0.01-0.02 pc.

One explanation might be that the gas observed here is denser than typical CNM gas. In the bright parts of the MegaCam field, the column density estimated from the radiance (using the conversion factor given by Planck Collaboration XI 2014) is $N_{\mathrm{H}}=3.1 \times 10^{21} \mathrm{~cm}^{-2}$. On the other hand the column density derived from $21 \mathrm{~cm}$ data peaks at $N_{\mathrm{HI}} \approx 4.6 \times 10^{20} \mathrm{~cm}^{-2}$. Part of this difference is due to the different angular resolution $\left(5^{\prime}\right.$ and $30^{\prime}$ ) but it can not explain it entirely. The difference between dust-derived and $\mathrm{H}$ I column density is an indication that a significant fraction of the gas is molecular, implying a higher density than usual CNM. There is indeed detectable ${ }^{12} \mathrm{CO}(J=2-1)$ emission in the Planck data at the position of the MegaCam field (Fig. 7). If the gas is denser $\left(n \sim 10^{3}-10^{4} \mathrm{~cm}^{-3}\right)$ and the amplitude of the magnetic field typical of the diffuse ISM $(B \sim 6 \mu \mathrm{G})$, it could explain why the dissipation scale is not visible in the power spectrum of the column density. Another potential explanation is that the cloud is further away than $200 \mathrm{pc}$.

\section{Conclusion}

We have presented a power spectrum analysis of the dust column density of a diffuse area at high Galactic latitude $\left(l \approx 198^{\circ}\right.$, $b \approx 32^{\circ}$ ) as derived from three different tracers: Planck radiance, WISE $12 \mu \mathrm{m}$ and MegaCam $g$ band. The combination of these three datasets allowed us to compute the density power spectrum of the CNM over more than three orders of magnitudes in scales. We found that the density field is well described as a

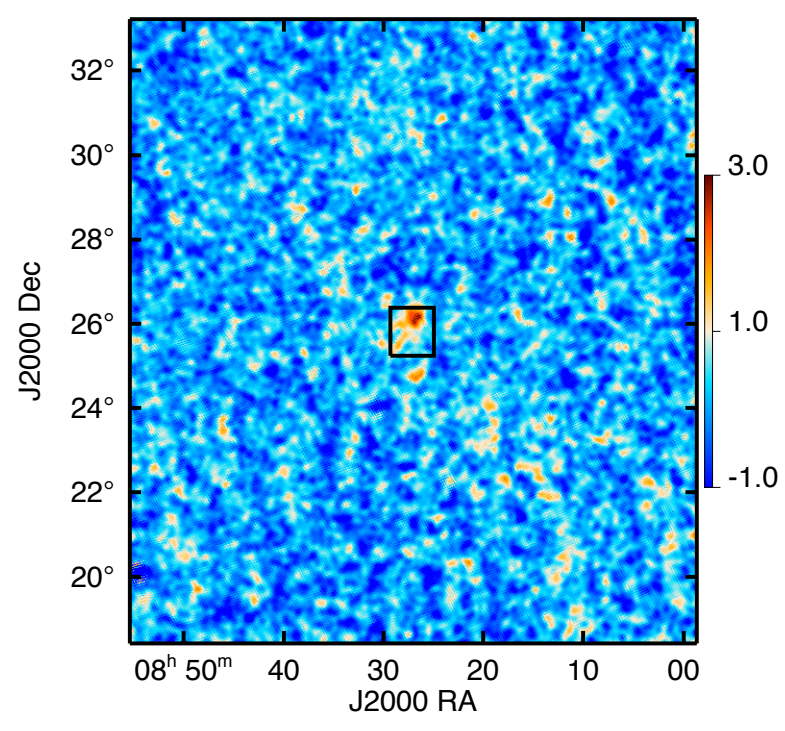

Fig. 7. Map of CO emission $(J=2-1)$ as observed by Planck. The map covers the same area as the Planck radiance (Fig. 1) and the $21 \mathrm{~cm}$ data (Fig. 5). There is no detected emission in the $J=1-0$ Planck map. Units are $K_{\mathrm{CMB}}$. The black square indicates the ATLAS field.

single power law over scales ranging from 0.01 to $50 \mathrm{pc}$. The power law exponent, $\gamma=-2.9 \pm 0.1$, is compatible with what is expected for the density field of thermally bi-stable and turbulent $\mathrm{H}$.

No significant flattening of the spectrum is observed at large scales, indicating that the energy injection scale is likely to be larger than $50 \mathrm{pc}$. In addition, we did not find any steepening of the power spectrum at small scales indicating that the typical scale at which turbulent energy is dissipated in this medium is at scales smaller than $0.01 \mathrm{pc}$. The comparison of the dust tracers with $21 \mathrm{~cm}$ and $\mathrm{CO}$ data is indicative of a CNM density slightly larger than average. In this case, the ambipolar diffusion scale is likely to be smaller than $0.01 \mathrm{pc}$.

We suggest that optical observations of diffuse high Galactic latitude regions can be used as a tracer of the properties of interstellar turbulence at small angular scale. In good seeing conditions, observations with MegaCAM on the CFH telescope can provide images at a resolution up to $0.5 \mathrm{arcsec}$. For clouds at a typical distance of $100 \mathrm{pc}$, this angular resolution corresponds to a linear physical scale of $2 \times 10^{-4} \mathrm{pc}$, or about $50 \mathrm{AU}$. The field used in this analysis has a rather poor seeing but was selected because of its strong and large scale cirrus distribution that allowed us to test the feasibility of the method. The MATLAS large program already provides a high number of images contaminated by the DGL. It is present in about $20 \%$ of the 241 fields observed at all Galactic latitudes with seeing conditions between 0.5 and 1.7 arcsec in the $g$ band. While cirrus emission has a deterrent impact on extragalactic studies - its patchy structure is indeed difficult to subtract -, it offers a powerful new tool to investigate the structure of the diffuse ISM and constrain the process of energy dissipation. With this database, we can probe a variety of cloud densities and distances and most likely reach scales below $0.01 \mathrm{pc}$. In the future, the Euclid mission will map a large part of the sky at the unprecedented resolution of 0.1 arcsec, possibly offering an unexpected new avenue for the study of the DGL.

Acknowledgements. We thank the referee, Paul Goldsmith, for his careful reading of the manuscript. The paper is based on observations obtained with 
MegaPrime/MegaCam, a joint project of CFHT and CEA/IRFU, at the CanadaFrance-Hawaii Telescope (CFHT) which is operated by the National Research Council (NRC) of Canada, the Institut National des Sciences de l'Univers of the Centre National de la Recherche Scientifique (CNRS) of France, and the University of Hawaii. The paper is also based on data from Planck, a project of the European Space Agency (ESA) with instruments provided by two scientific consortia funded by ESA member states (in particular lead countries France and Italy), with contributions from NASA (USA) and telescope reflectors provided by a collaboration between ESA and a scientific consortium led and funded by Denmark. The paper is also based on data from WISE, the Wide-field Infrared Survey Explorer, a joint project of the University of California, Los Angeles, and the Jet Propulsion Laboratory/California Institute of Technology, funded by the National Aeronautics and Space Administration.

\section{References}

Arzoumanian, D., André, P., Didelon, P., et al. 2011, A\&A, 529, L6

Barnard, E. E. 1910, ApJ, 31, 8

Beck, R. 2001, Space Sci. Rev., 99, 243

Bergin, E. A., \& Tafalla, M. 2007, ARA\&A, 45, 339

Bertin, E., \& Arnouts, S. 1996, A\&AS, 117, 393

Blagrave, K., Martin, P. G., Joncas, G., et al. 2016, ApJ, submitted [arXiv: 1602.02115]

Boissier, S., Boselli, A., Voyer, E., et al. 2015, A\&A, 579, A29

Brandt, T. D., \& Draine, B. T. 2012, ApJ, 744, 129

Cappellari, M., Emsellem, E., Krajnović, D., et al. 2011, MNRAS, 413, 813

Cortese, L., Bendo, G. J., Isaak, K. G., Davies, J. I., \& Kent, B. R. 2010 MNRAS, 403, L26

Crutcher, R. M. 1999, ApJ, 520, 706

de Vaucouleurs, G. 1955, AJ, 60, 126

de Vaucouleurs, G. 1960, The Observatory, 80, 106

de Vries, C. P. \& Le Poole, R. S. 1985, A\&A, 145, L7

Dickey, J. M., \& Lockman, F. J. 1990, ARA\&A, 28, 215

Duc, P.-A., Cuillandre, J.-C., Karabal, E., et al. 2015, MNRAS, 446, 120

Elvey, C. T., \& Roach, F. E. 1937, ApJ, 85, 213

Ferrarese, L., Côté, P., Cuillandre, J.-C., et al. 2012, ApJS, 200, 4

Ferrière, K. M. 2001, Rev. Mod. Phys., 73, 1031

Fischera, J., \& Martin, P. G. 2012, A\&A, 542, A77

Gautier, III, T. N., Boulanger, F., Perault, M., \& Puget, J. L. 1992, AJ, 103, 1313

Gazol, A., \& Kim, J. 2010, ApJ, 723, 482

Guhathakurta, P., \& Tyson, J. A. 1989, ApJ, 346, 773

Heiles, C., \& Crutcher, R. 2005, in Cosmic Magnetic Fields, Lect. Notes Phys., 664,137

Hennebelle, P. 2013, A\&A, 556, A153
Hennebelle, P., \& André, P. 2013, A\&A, 560, A68

Ienaka, N., Kawara, K., Matsuoka, Y., et al. 2013, ApJ, 767, 80

Juvela, M., Pelkonen, V.-M., Padoan, P., \& Mattila, K. 2006, A\&A, 457, 877

Kalberla, P. M. W., Burton, W. B., Hartmann, D., et al. 2005, A\&A, 440, 775

Kapteyn, J. C. 1909, ApJ, 29, 46

Kim, J., \& Ryu, D. 2005, ApJ, 630, L45

Kritsuk, A. G., Norman, M. L., Padoan, P., \& Wagner, R. 2007, ApJ, 665, 416

Lallement, R., Vergely, J.-L., Valette, B., et al. 2014, A\&A, 561, A91

Laureijs, R. J., Mattila, K., \& Schnur, G. 1987, A\&A, 184, 269

Lequeux, J. 2005, The Interstellar Medium (CNRS Édition)

Low, F. J., Young, E., Beintema, D. A., et al. 1984, ApJ, 278, L19

Lynds, B. T. 1965, ApJS, 12, 163

Malinen, J., Juvela, M., Rawlings, M. G., et al. 2012, A\&A, 544, A50

Malinen, J., Juvela, M., Pelkonen, V.-M., \& Rawlings, M. G. 2013, A\&A, 558, A44

Martin, P. G., Blagrave, K. P. M., Lockman, F. J., et al. 2015, ApJ, 809, 153

Martínez-Delgado, D., Gabany, R. J., Crawford, K., et al. 2010, AJ, 140, 962

Meisner, A. M., \& Finkbeiner, D. P. 2014, ApJ, 781, 5

Miville-Deschênes, M.-A., Boulanger, F., Joncas, G., \& Falgarone, E. 2002a, A\&A, 381, 209

Miville-Deschênes, M.-A., Lagache, G., \& Puget, J.-L. 2002b, A\&A, 393, 749

Miville-Deschênes, M.-A., Levrier, F., \& Falgarone, E. 2003, ApJ, 593, 831

Miville-Deschênes, M.-A., Lagache, G., Boulanger, F., \& Puget, J.-L. 2007, A\&A, 469, 595

Miville-Deschênes, M.-A., Martin, P. G., Abergel, A., et al. 2010, A\&A, 518, L104

Miville-Deschênes, M.-A., Salomé, Q., Martin, P. G., et al. 2016, A\&A, submitted [arXiv: 1602.03340]

Ntormousi, E., Hennebelle, P., André, P., \& Masson, J. 2016, A\&A, 589, A24

Paley, E. S., Low, F. J., McGraw, J. T., Cutri, R. M., \& Rix, H.-W. 1991, ApJ, 376,335

Planck Collaboration XI. 2014, A\&A, 571, A11

Planck Collaboration Int. XIX. 2015, A\&A, 576, A104

Planck Collaboration Int. XX. 2015, A\&A, 576, A105

Planck Collaboration Int. XXXII. 2016, A\&A, 586, A135

Planck Collaboration Int. XXXIII. 2016, A\&A, 586, A136

Planck Collaboration Int. XXXV. 2016, A\&A, 586, A138

Sandage, A. 1976, AJ, 81, 954

Saury, E., Miville-Deschênes, M.-A., Hennebelle, P., Audit, E., \& Schmidt, W. 2014, A\&A, 567, A16

Soler, J. D., Hennebelle, P., Martin, P. G., et al. 2013, ApJ, 774, 128

Stepnik, B., Abergel, A., Bernard, J.-P., et al. 2003, A\&A, 398, 551

Wolfire, M. G., McKee, C. F., Hollenbach, D., \& Tielens, A. G. G. M. 2003, ApJ, 587,278

Zagury, F., Boulanger, F., \& Banchet, V. 1999, A\&A, 352, 645 